\title{
Vestibulo-Ocular Reflex Suppression during Head-Fixed Saccades Reveals Gaze Feedback Control
}

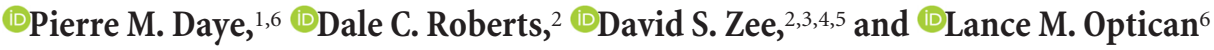 \\ ${ }^{1}$ Pierre et Marie Curie Paris-6 Université, INSERM UMRS 975, CNRS 7225, Paris, France, Departments of ${ }^{2}$ Neurology, ${ }^{3}$ Otolaryngology, ${ }^{4}$ Ophthalmology, \\ and ${ }^{5}$ Neuroscience, The Johns Hopkins University, School of Medicine, Baltimore, Maryland 21287, and ${ }^{2}$ Laboratory of Sensorimotor Research, National \\ Eye Institute, National Institutes of Health, Bethesda, Maryland 20892-4435
}

Previous experiments have shown that the vestibulo-ocular reflex (VOR) is partially suppressed during large head-free gaze (gaze $=$ eye-in-head + head-in-space) shifts when both the eyes and head are moving actively, on a fixed body, or when the eyes are moving actively and the head passively on a fixed body. We tested, in human subjects, the hypothesis that the VOR is also suppressed during gaze saccades made with en bloc, head and body together, rotations. Subjects made saccades by following a target light. During some trials, the chair rotated so as to move the entire body passively before, during, or after a saccade. The modulation of the VOR was a function of both saccade amplitude and the time of the head perturbation relative to saccade onset. Despite the perturbation, gaze remained accurate. Thus, VOR modulation is similar when gaze changes are programmed for the eyes alone or for the eyes and head moving together. We propose that the brain always programs a change in gaze using feedback based on gaze and head signals, rather than on separate eye and head trajectories.

Key words: eye-head saccades; feedback control; VOR

\section{Introduction}

We constantly redirect our gaze (the position of the eye relative to our external environment) from one center of interest to another with coordinated eye and head movements. Along with these desired gaze movements, life's activities (e.g., walking; Grossman et al., 1988) also generate unplanned head movements. To maintain clear vision something must compensate for those perturbations of the head. The vestibulo-ocular reflex (VOR) is a short latency response that counter-rotates the eyes so that the line of sight stays stable during unanticipated head movements. In some circumstances, the VOR can be counterproductive, as when a large change in gaze requires that gaze and the head move in the same direction. During active head movements, two mechanisms (cancellation and suppression) have been proposed to prevent interference from the VOR. The cancellation mechanism generates a command opposite to the head command to negate the action of the VOR, e.g., during pursuit (Lanman et al., 1978). During large saccades the suppression mechanism decreases the VOR gain at the onset of a head-unrestrained saccade and in-

\footnotetext{
Received Sept. 18, 2014; revised Nov. 12, 2014; accepted Nov. 16, 2014.

Author contributions: P.M.D., D.S.Z., and L.M.O. designed research;P.M.D., D.C.R., D.S.Z., and L.M.O. performed research; P.M.D. contributed unpublished reagents/analytic tools; P.M.D. and L.M.O. analyzed data; P.M.D., D.S.Z., and L.M.O. wrote the paper.

D.C.R. was supported by Betty and Paul Cinquegrana, P.M.D. and L.M.O. were supported by the intramural research program of the National Eye Institute of the National Institutes of Health, and P.M.D. was supported by le Fond de La Recherche Médicale and the CERVISO program. D.S.Z. was supported by the Lott family foundation.

The authors declare no competing financial interests.

Correspondence should be addressed to Dr Pierre M. Daye, Pierre et Marie Curie Paris-6 Université, INSERM UMRS 975, CNRS 7225, Paris, France. E-mail: pierre.daye@gmail.com.

DOI:10.1523/JNEUROSCI.3875-14.2015

Copyright $\odot 2015$ the authors $\quad 0270-6474 / 15 / 351192-07 \$ 15.00 / 0$
}

creases it back toward one before the end of the saccade (Tomlinson and Bahra, 1986; Lefèvre et al., 1992; Cullen et al., 2004). Here we address only the suppression mechanism. Traditionally, the suppression mechanism has been studied during combined eye-head saccades when the head is free to move and is perturbed by long-lasting torques opposing or assisting the head movement (Boulanger et al., 2012), short torque pulses opposing or assisting the head movement (Laurutis and Robinson, 1986; Tomlinson and Bahra, 1986; Cullen et al., 2004), when the head is immobilized by a brake for 50-400 ms (Guitton and Volle, 1987), or by comparing oscillating and pulsed perturbations (Tabak et al., 1996). Others have compared saccadic trajectories under headfixed and head-free conditions (Lefèvre et al., 1992) or with passive head-on-body rotations (assisting or opposing the head movement; Pelisson et al., 1988). Our study fills an important gap in our understanding of eye-head coordination by combining these two approaches to compare gaze trajectories with and without whole-body rotations during saccades that are made when the head and body are fixed with respect to the chair. We found that the time course of the change of the gaze trajectory, relative to a head perturbation, is similar to the time course reported previously when the head is perturbed during head-free saccades (Laurutis and Robinson, 1986; Tomlinson and Bahra, 1986; Cullen et al., 2004). Importantly, we also found that gaze remained accurate despite the head perturbations, even though the VOR was suppressed. These findings are compatible with a series of models that suggest that the brain uses feedback control of gaze trajectory, and not eye trajectory to change the line of sight (Laurutis and Robinson, 1986; Guitton and Volle, 1987; Pelisson et al., 1988; Guitton et al., 1990; Tomlinson, 1990; Lefèvre and Galiana, 1992; Goossens and Van Opstal, 1997; Daye et al., 2014). Our 
results imply further that the passively induced VOR is modulated by a gaze rather than an eye velocity signal.

\section{Materials and Methods}

Subjects. Three male subjects (S1, S2, and S3, aged 32-69 years) were recorded in the experiment; none of them had any known oculomotor or visual abnormalities. Two authors were subjects for the study (S1 and S3), whereas the third subject was naive about the purpose of the study (S2). The study was approved by The Johns Hopkins University Institutional Review Board and was in compliance with the Declaration of Helsinki. All subjects gave informed consent.

Experimental setup. Human participants sat on a motorized rotary chair facing a row of nine red LEDs located $189 \mathrm{~cm}$ away in an otherwise darkened room. Eight LEDs spanned $\pm 20^{\circ}$ of the horizontal visual arc by steps of $5^{\circ}$ around a central fixation LED. The subject's head was firmly clamped to the chair to reduce any relative movement between the head and chair. Software controlled the presentation of the targets and the motion of the chair. Horizontal eye movements were recorded monocularly using the magnetic field search-coil technique. Eye drops were provided, as needed, for comfort. A second coil was taped to the subjects' forehead to record head movements. Finally, a third coil was fixed to the chair to record its rotation. All signals were recorded at $1 \mathrm{kHz}$. The axis of chair rotation was $\sim 3 \mathrm{~cm}$ behind the center of the otoliths (which are $\sim 7$ $\mathrm{cm}$ behind the eyes; Crane et al., 1997). Thus, motion of the chair induced both a rotation and a translation of the orbits, causing both a rotational VOR (rVOR) and a translational VOR (tVOR). The tVOR, however, is a function of the reciprocal of the distance to the target, which was quite far in our experiment (Schwarz et al., 1989; Snyder and King, 1992; Crane et al., 1997). Thus, we treat the eye movements as if they were due only to the rVOR.

Calibration. We performed one calibration at the beginning of the experiment. A calibration consisted of 13 sequences of nine fixations (each fixation lasted $1 \mathrm{~s}$ ). During each visual target sequence, the chair remained fixed. Between two target sequences the chair moved to a new position according to its own sequence (chair position $0,4,8,12,8,4,0$, $-4,-8,-12,-8,-4$, and $\left.0^{\circ}\right)$. Subjects were required to look at the targets when they came on.

Experimental design. Each subject was recorded under two conditions. In both conditions, a trial started when the subject looked at the central target. After a random duration $(800 \ldots 1000 \mathrm{~ms})$, the central target was extinguished and concurrently a lateral target was presented randomly either at $-20,-10,10$ or $20^{\circ}$ for a random duration $(800 \ldots 1000 \mathrm{~ms})$. Then the target was extinguished and the symmetrical target in the opposite direction was switched on for a random duration $(800 \ldots 1000 \mathrm{~ms})$. The trial ended with another central fixation. In the first condition (control condition), the chair remained fixed during all the trials. In the second condition (perturbed condition), the chair rotated $4^{\circ}$ to the left or to the right, with a brief triangular velocity pulse (peak acceleration: $1500^{\circ} / \mathrm{s}^{2}$ ). The onset of chair motion was triggered randomly 110,125 , 150,160 or $200 \mathrm{~ms}$ after the jump to the second target. At the end of a trial, the chair rotated back toward the center. Each trial contained a perturbation in the second block. Therefore, subjects knew that a perturbation would occur but they could predict neither the direction nor the timing of the perturbation. Finally, to add some variability in the initial eye-in-head position, the starting position of the chair was set to 0,4 , or $-4^{\circ}$ with respect to the central fixation target. All the trial sequences were computed offline. Therefore, each subject had the same number of trials for each condition. A block of trials did not exceed $5 \mathrm{~min}$.

Data analyses. Data were stored for offline analysis. All the analyses were performed on a personal computer with custom software written in the python programming language. Signals from the three coils (gaze, head, and chair) were low-pass filtered using a third-order zero-phase infinite impulse response digital filter (cutoff frequency: $50 \mathrm{~Hz}$, filter length $10 \mathrm{~ms}$ ). Velocity and acceleration were derived using a centraldifference algorithm (moving window: $10 \mathrm{~ms}$ ). Data were normalized such that the symmetrical target jumps always appeared to be rightward.

Saccade detection. Gaze saccades were detected using a $20 \%$ s velocity threshold. Chair and head movement onset were detected with $5 \%$ selocity thresholds.
A
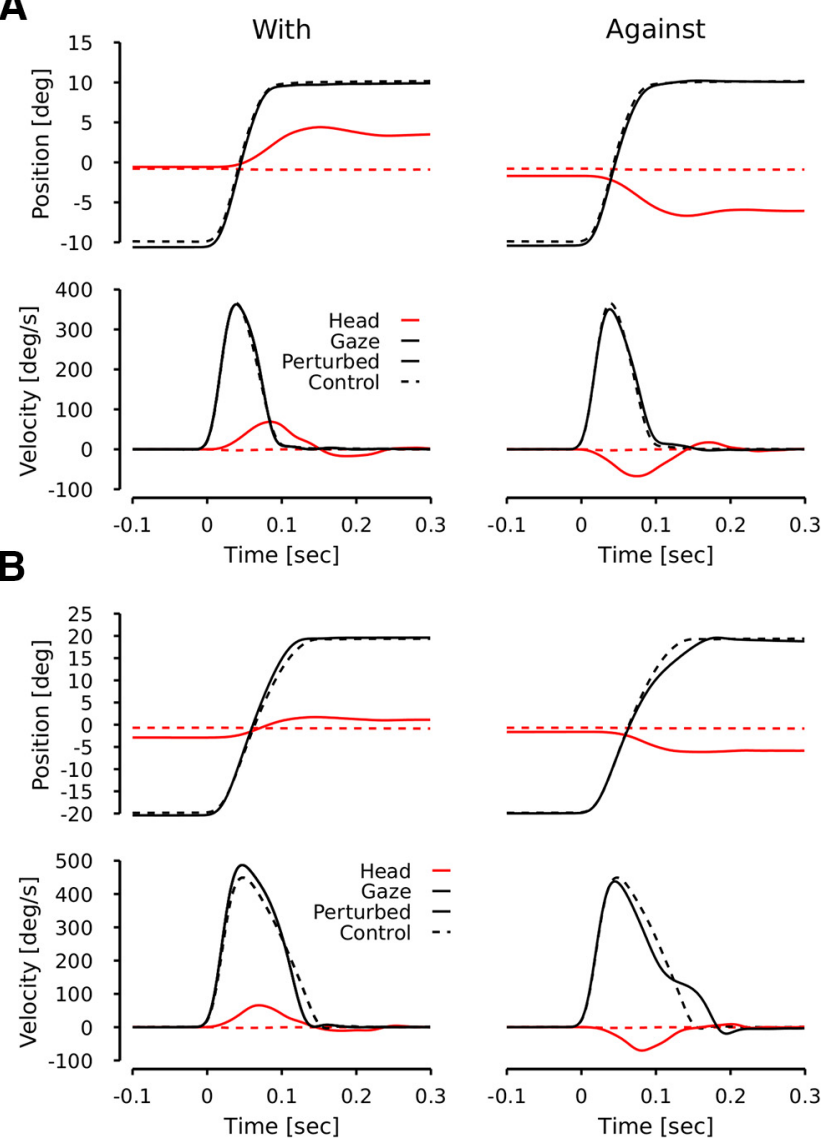

Figure 1. Typical examples. $\boldsymbol{A}, \mathrm{A}$ typical case for a $20^{\circ}\left(40^{\circ}, \boldsymbol{B}\right)$ target jump for $\mathrm{S1}$. In both panels, the top row represents the time course of position signals (red lines for head, black lines for gaze). The bottom row represents the time course of velocity signals. Dashed lines represent the control condition. Solid lines represent the perturbed condition. Left (right) column represents perturbed cases when the chair moved with (against) the gaze movement.

Dataset. We recorded two control blocks and 10 perturbed blocks for each subject. We collected 10,237 trials of which 7202 were valid (70.2\%). Trials were rejected when saccade latency was $<50 \mathrm{~ms}(20.4 \%)$ or $>300$ $\mathrm{ms}(0.3 \%)$, if the gaze was moving when the target jumped (5.5\%), if the peak gaze acceleration was smaller than $7000^{\circ} / \mathrm{s}^{2}\left(10,000^{\circ} / \mathrm{s}^{2}\right)$ for $20^{\circ}$ $\left(40^{\circ}\right)$ target jumps $(1.8 \%)$, if the head slipped $(0.8 \%)$, if the saccade gain was lower than $0.7(0.4 \%)$ or if the subject blinked after the target jumped $(0.6 \%)$. Statistical comparisons were made with resampling techniques. Sample sizes of the control and the perturbed conditions were very different. To account for this, samples were randomly selected without replacement in the larger dataset and with replacement in the smaller dataset (bootstrap $N=30^{\star}$ smaller sample size; Good, 2006).

\section{Results}

\section{Typical trials}

Figure 1 shows typical trials for $20^{\circ}(A)$ and $40^{\circ}(B)$ target jumps. Control conditions are represented by dashed lines. Solid lines represent perturbed conditions. Figure 1, the left (right) column in both panels show perturbed examples when the chair moved in the same (opposite) direction as the gaze. The head started to move $26 \mathrm{~ms}$ (12 ms) later than the gaze in the left (right) column of Figure $1 A$. The head started to move $41 \mathrm{~ms}$ ( $33 \mathrm{~ms}$ ) later than the gaze in the left (right) column of Figure $1 B$. Figure 1 shows that the head movement noticeably changed the gaze trajectory for $40^{\circ}$ target jumps, but only slightly affected the gaze trajectory for $20^{\circ}$ target jumps. This is consistent with a VOR suppression mechanism modulated by the amplitude of the planned gaze 
Table 1. Difference between gaze position $15 \mathrm{~ms}$ after gaze saccade offset between the control and the perturbed conditions as a function of relative latency (in $20 \mathrm{~ms}$ bins centered on $\Delta \mathrm{L}$ )

\begin{tabular}{|c|c|c|c|c|c|c|c|c|c|c|c|c|c|c|c|c|c|c|c|}
\hline \multirow[b]{2}{*}{ Subject } & \multirow{2}{*}{$\frac{\Delta \mathrm{L}(\mathrm{s})}{\mathrm{T} \text { jump }}$} & \multicolumn{2}{|l|}{-0.12} & \multicolumn{2}{|l|}{-0.10} & \multicolumn{2}{|l|}{-0.8} & \multicolumn{2}{|l|}{-0.6} & \multicolumn{2}{|l|}{-0.4} & \multicolumn{2}{|l|}{-0.2} & \multicolumn{2}{|l|}{0.0} & \multicolumn{2}{|l|}{0.2} & \multicolumn{2}{|l|}{0.4} \\
\hline & & A & W & A & W & A & W & A & W & A & W & A & W & A & W & A & W & $A$ & W \\
\hline \multirow[t]{2}{*}{ S1 } & 20 & $-0.43^{* * *}$ & -0.41 & 0.09 & 0.09 & -0.32 & 0.14 & 0.03 & -0.15 & -0.07 & -0.14 & -0.22 & -0.04 & 0.08 & $-0.18^{*}$ & - & - & - & - \\
\hline & 40 & -0.04 & -0.09 & - & - & $-0.63^{* *}$ & $-0.51^{*}$ & -0.38 & -0.06 & -0.15 & -0.04 & -0.15 & $-0.4^{*}$ & -0.61 & -0.23 & - & - & - & - \\
\hline \multirow[t]{2}{*}{ S2 } & 20 & - & - & - & - & -0.12 & 0.00 & 0.00 & -0.02 & -0.03 & -0.15 & -0.05 & 0.05 & -0.10 & $-0.14^{*}$ & - & - & - & - \\
\hline & 40 & - & - & - & - & $-0.27^{* * *}$ & -0.04 & -0.13 & -0.13 & 0.24 & -0.14 & -0.15 & -0.12 & $-0.32^{*}$ & -0.17 & - & - & - & - \\
\hline \multirow[t]{2}{*}{ S3 } & 20 & - & - & -0.07 & 0.13 & -0.02 & -0.15 & -0.05 & -0.04 & -0.08 & -0.03 & -0.02 & -0.09 & - & - & - & - & - & - \\
\hline & 40 & - & - & -0.07 & $-0.19 *$ & -0.25 & -0.01 & 0.04 & 0.04 & -0.06 & -0.05 & $-0.18^{* * *}$ & -0.04 & 0.05 & -0.14 & - & - & - & - \\
\hline \multirow[t]{2}{*}{ All } & 20 & $-0.35^{* *}$ & -0.17 & -0.05 & $0.12^{*}$ & -0.08 & 0.01 & -0.01 & -0.05 & -0.06 & -0.07 & -0.07 & -0.05 & -0.06 & $-0.16^{* *}$ & -0.12 & -0.12 & - & - \\
\hline & 40 & -0.01 & -0.01 & -0.09 & -0.15 & $-0.40^{* * *}$ & -0.10 & -0.07 & 0.03 & 0.02 & -0.09 & $-0.18^{* *}$ & -0.12 & $-0.27^{* *}$ & -0.21 & -0.19 & $-0.34^{* *}$ & $-0.66^{* * *}$ & $-0.46^{* * *}$ \\
\hline
\end{tabular}

$\mathrm{A}$, The chair moved in the opposite direction to the gaze; $\mathrm{W}$, the chair moved in the same direction as the gaze (differences are expressed in degrees); $\Delta \mathrm{L}$, relative latency between gaze onset and head perturbation.

$-=$ Not enough points $(<10)$. Significantly different from $0:{ }^{*} p<0.05,{ }^{* *} p<0.01,{ }^{* * *} p<0.001$.

shift: for small saccades the VOR gain is $\sim 1$, and trajectories remain unaffected, whereas the VOR gain is $<1$ for large saccades, and trajectories are affected by the head perturbations (see Discussion for the ambiguity in computing the VOR gain). Importantly, despite the effect on gaze trajectories caused by the head movement for the $40^{\circ}$ saccades, the final gaze position of the perturbed cases remained close to the control position. This shows that the gaze controller compensates for the perturbation, even though the VOR is suppressed. Our results are thus similar to previous observations made in head-free conditions when subjects performed a combined active eye-head gaze shift during which a transient external perturbation was applied to the head (Laurutis and Robinson, 1986; Tabak et al., 1996; Tomlinson and Bahra, 1986; Cullen et al., 2004; Boulanger et al., 2012).

\section{Gaze accuracy}

We compared the gaze position shortly after saccade offset between the control and the perturbed conditions as a function of the relative latency between the onset of the chair-induced head movement and the saccade onset $(\Delta \mathrm{L}=$ gaze onset - head onset). $\Delta \mathrm{L}<0(\Delta \mathrm{L}>0)$ represents a head movement that started after (before) gaze onset. To test whether the perturbation changed the gaze accuracy, we bootstrapped the difference between gaze position of control and perturbed conditions averaged between 10 and $20 \mathrm{~ms}$ after saccade offset. Table 1 shows the position difference at saccade offset for the three subjects and for all the subjects pooled together. The differences in Table 1 are not statistically significant for most conditions. In addition, even when there was a statistically significant difference from zero, the relative difference (difference divided by the amplitude of the target jump) was lower than $\sim 2 \%$. This analysis demonstrates that, despite the perturbation, the combined action of the VOR and gaze command compensated for the head displacement to ensure that gaze ended on the target.

\section{Time course of the modulation of the gaze trajectory induced by a perturbation}

Figure 2 represents the evolution of the difference in gaze velocity $(V)$ between the control and the perturbed situations for three different ranges of $\Delta \mathrm{L}$ values. We divided the dataset into bins of $\Delta \mathrm{L}$ (bin size $=20 \mathrm{~ms}$ ). When a bin contained $>10$ trials, we bootstrapped the computation of the median trajectory. Then we computed the median (Fig. 2, thick lines) of the bootstrapped distribution as well as the $95 \%$ confidence interval (Fig. 2, thin lines). This was done separately for both the control and the perturbed conditions. We also bootstrapped the median of the difference between the control and the perturbed conditions, $d V$ (Fig. 2, black lines).
Figure 2 shows that the difference $(\mathrm{d} V)$ between the median velocity trajectories is not statistically significant before the onset of the head movement (the $95 \%$ confidence interval of $\mathrm{d} V$ contains 0 ). Figure 2 also shows that the sooner the perturbation occurred with respect to the gaze onset, the larger the difference between the perturbed and the control situations. Comparing the amplitude of the black lines, one can see that the head movement affected the gaze trajectory more drastically when both movements started at the same time $(\Delta \mathrm{L}=0$, right column $)$ compared to when the chair moved after the gaze shift began $(\Delta \mathrm{L}<0$, left column). In addition, $d V$ amplitude increased as a function of the amplitude of the target jump. If we regard $d V$ as biphasic, the initial phase follows the head movement, which is consistent with the VOR having a gain less than one. The second phase of $\mathrm{d} V$ is sensitive to the relative head direction with respect to the gaze. When the head opposed the gaze movement, the amplitude of $\mathrm{d} V$ was larger (the gaze controller had to accelerate the movement to reach the target) than when the head moved in the same direction as the gaze (the gaze got closer to the target faster, so the gaze controller provided less drive to reach the target). This is consistent with the second phase reflecting the output of a gaze feedback controller. The sensitivity of $\mathrm{d} V$ to the amplitude of the desired gaze shift and to the relative latency between the gaze and the target onset in our head-fixed experiment agrees with the head-free saccadic experiments done by Cullen et al. (2004).

\section{Evolution of the amplitude of the modulation of gaze trajectory as a function of the relative onset of head movement with respect to gaze onset}

To quantify the observations shown in Figure 2, we first computed the ratio $\left(G P_{\max }\right)$ of $\mathrm{d} V$ at the peak head velocity to the peak head velocity. Then we plotted $G P_{\max }$ as a function of the relative onset of the head movement with respect to the onset of the gaze change (relative onset of head motion; $\mathrm{ROHM}=\Delta \mathrm{L} / \mathrm{sac}$ cade duration). A GP $P_{\max }$ equal to 0 means that there is no difference between the perturbed and the control conditions. When $G P_{\max }=1, \mathrm{~d} V$ is equal to the peak chair velocity (no compensation of the head movement). When $G P_{\max }<0$, there is an overcompensation of the head movement because the gaze moved in the opposite direction to the head. Overcompensation has been described previously and attributed to the latency of the VOR response (Collewijn and Smeets, 2000). The value of $G P_{\max }$ was bootstrapped if there were at least 10 samples in a bin. Figure 3 shows $G P_{\max }$ as a function of ROHM for each subject (colored lines) and for all the data pooled together (black lines). The upper (lower) row represents the $20^{\circ}\left(40^{\circ}\right)$ target jumps. The left (right) column represents perturbed cases when the head moved in the same (opposite) direction as the gaze. Figure 3 shows that for 

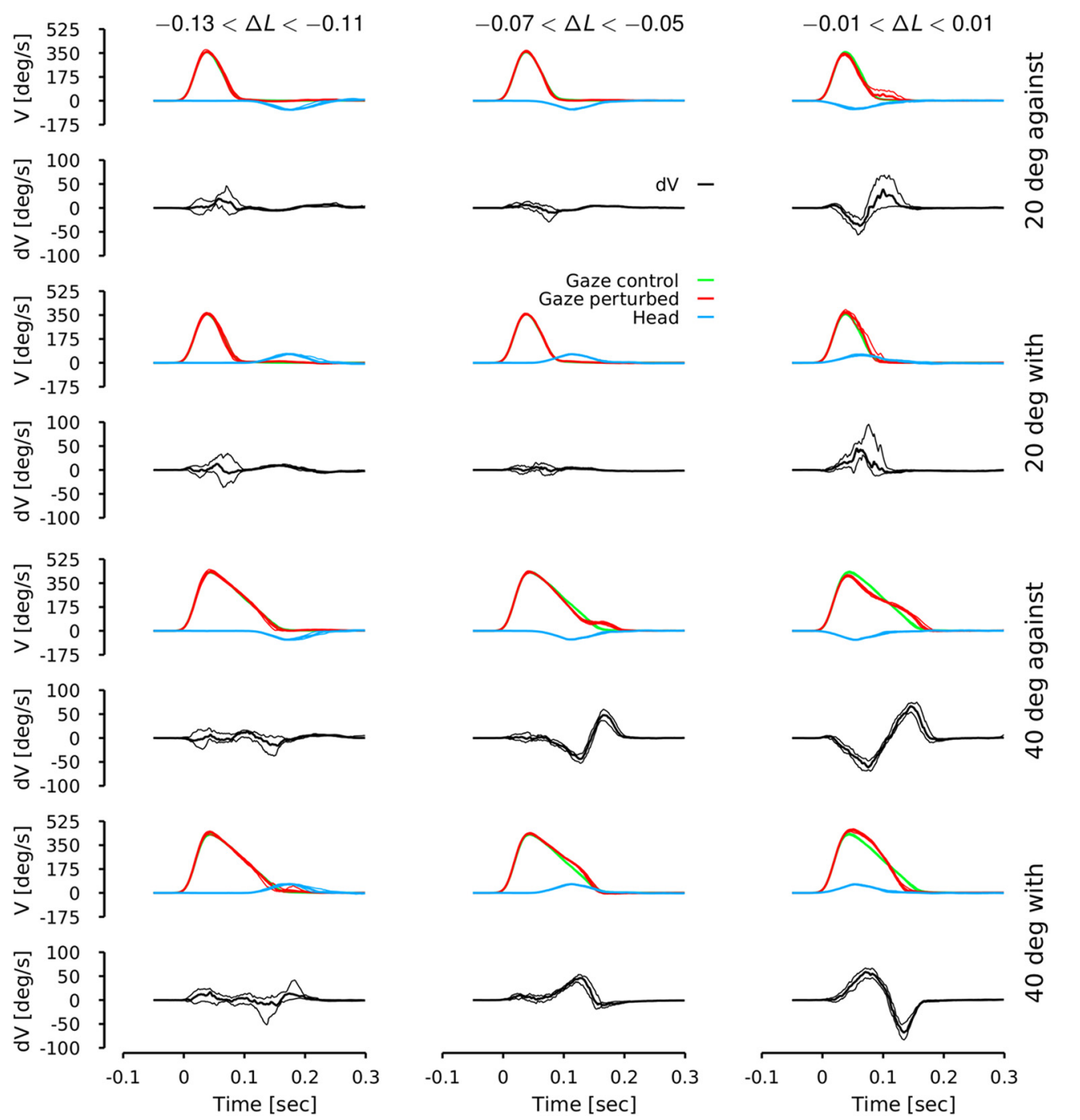

Figure 2. Averaged velocity $(\mathrm{V})$ trajectories as a function of the relative chair latency $(\Delta \mathrm{L})$, the direction of the perturbation and the amplitude of the target jump. $\Delta \mathrm{L}$ is the difference between gaze onset and head onset. Green lines represent the control gaze velocity. Red lines represent the perturbed gaze velocity. Cyan lines represent the chair velocity. Black lines represent the difference between the control and the perturbed gaze velocity ( $\mathrm{dV}$ ). Thick lines represent the median of the different signals and thin lines represent the $95 \%$ confidence interval of the median of each signal. Behavior is shown when the target jump was equal to $20^{\circ}$ (top four rows) and $40^{\circ}$ (bottom row), and the chair moved with or against the gaze movement. All data from all subjects were pooled.

small target jumps, the gaze trajectory was little changed by the perturbation if it occurred after the onset of the gaze $(\mathrm{ROHM}<$ $0)$. At the time of the gaze onset, there was a significant modification of the gaze trajectory in both directions for S1 and only when the head went in the same direction as the gaze for S2 (we did not have enough data points collected at this latency to compute $G P_{\max }$ at saccade onset for $\mathrm{S} 3$ ). When the data from all the subjects were pooled together, a similar trend was observed at the onset of the gaze, independently of the direction of the target motion. When the head movement occurred before the onset of the gaze $(\mathrm{ROHM}>0), G P_{\max }$ decreased.

For large target jumps, an inverted $U$ shape characterizes $G P_{\max }$ as a function of ROHM. For large positive and large negative values of ROHM, the head motion had no significant effect on the gaze trajectory. In between, $G P_{\max }$ was uniformly high indicating an inadequate compensation for the head movement perturbation. A similar trend is present regardless of the perturbation direction (with or against). All the subjects followed the same rule. However, S2 had more variation (compare the size of the confidence intervals for S2 with those for S1 and S3).
Finally, we checked if saccade latency affected $G P_{\max }$ as a function of ROHM. We divided the dataset into two parts with respect to the median of the saccade latency distribution $(110 \mathrm{~ms})$. Figure 4 represents the evolution of $G P_{\max }$ as a function of ROHM for short (orange lines, latency $<110 \mathrm{~ms}$ ) and long (purple lines, latency $>110 \mathrm{~ms}$ ) saccade latency and for all the data pooled together (gray area: 95\% confidence interval). Figure 4 shows that the latency had no effect on the modulation of the $G P_{\max }$ as a function of ROHM (orange and purple lines are within the $95 \%$ confidence interval for all latencies) demonstrating that the critical parameters for this modulation are the relative onset of the perturbation with respect to saccade onset.

\section{Discussion}

This study analyzed how an unexpected, transient, passive movement of the head during saccades that were initiated while the head was fixed, affects the trajectory of saccades. We showed that the accuracy of the saccade was not altered by the head perturbation. A system based on separate eye and head controllers would not maintain accuracy, because the eye controller would have no 
way to assess the amount of the gaze trajectory that had been affected by a perturbation with a VOR gain $<1$. Thus, our results imply that the brain combines the action of the VOR (whatever its gain) and a feedback mechanism for gaze. We also showed that the saccade trajectory is affected more by the perturbation for larger saccade amplitudes, and when the onset of the head perturbation was close to the onset of the saccade. Finally, we showed that the modulation of the saccade trajectory was insensitive to the latency of the saccade with respect to the target presentation.

In our experiment, we created a passive rotation of the head with a peak velocity smaller than the saturation limit of the VOR, which is $\sim 350 \%$ s (Pulaski et al., 1981). Therefore, the modulation of the gaze trajectory generated by the head movement could not be attributed to a saturation of the ability of the VOR to compensate for high velocity rotations of the head. Thus, our results demonstrate that the VOR can be modulated when saccades are programmed in a head-fixed condition. The VOR modulation we found is similar to the VOR modulation in head-free experiments following head perturbations (Laurutis and Robinson, 1986; Tomlinson and Bahra, 1986; Guitton and Volle, 1987; Pelisson et al., 1988; Cullen et al., 2004). This result extends the observations made in the headunrestrained condition by Cullen et al. (2004) to saccades planned with the head fixed.

When the trajectory of large saccades was altered by the head perturbation, the VOR must have had a gain less than one (otherwise control and perturbed trajectories would be identical). Despite the modification of the saccade trajectory, the gaze ended on target, demonstrating feedback control that compensates for the perturbation. Two possibilities have been proposed for the control of head-free saccades: either the CNS controls the gaze trajectory with feedback (Laurutis and Robinson, 1986; Guitton and Volle, 1987; Pelisson et al., 1988; Guitton et al., 1990; Tomlinson, 1990; Lefèvre and Galiana, 1992; Goossens and Van Opstal, 1997; Daye et al., 2014), or it controls the eye and the head separately without gaze feedback (Freedman, 2001, 2008; Freedman and Cecala, 2008; Kardamakis and Moschovakis, 2009; Kardamakis et al., 2010). A separate control of eye and head based on a prior decomposition of the gaze command, with no feedback of the gaze and an imperfect VOR, could not compensate for the head perturbation be-

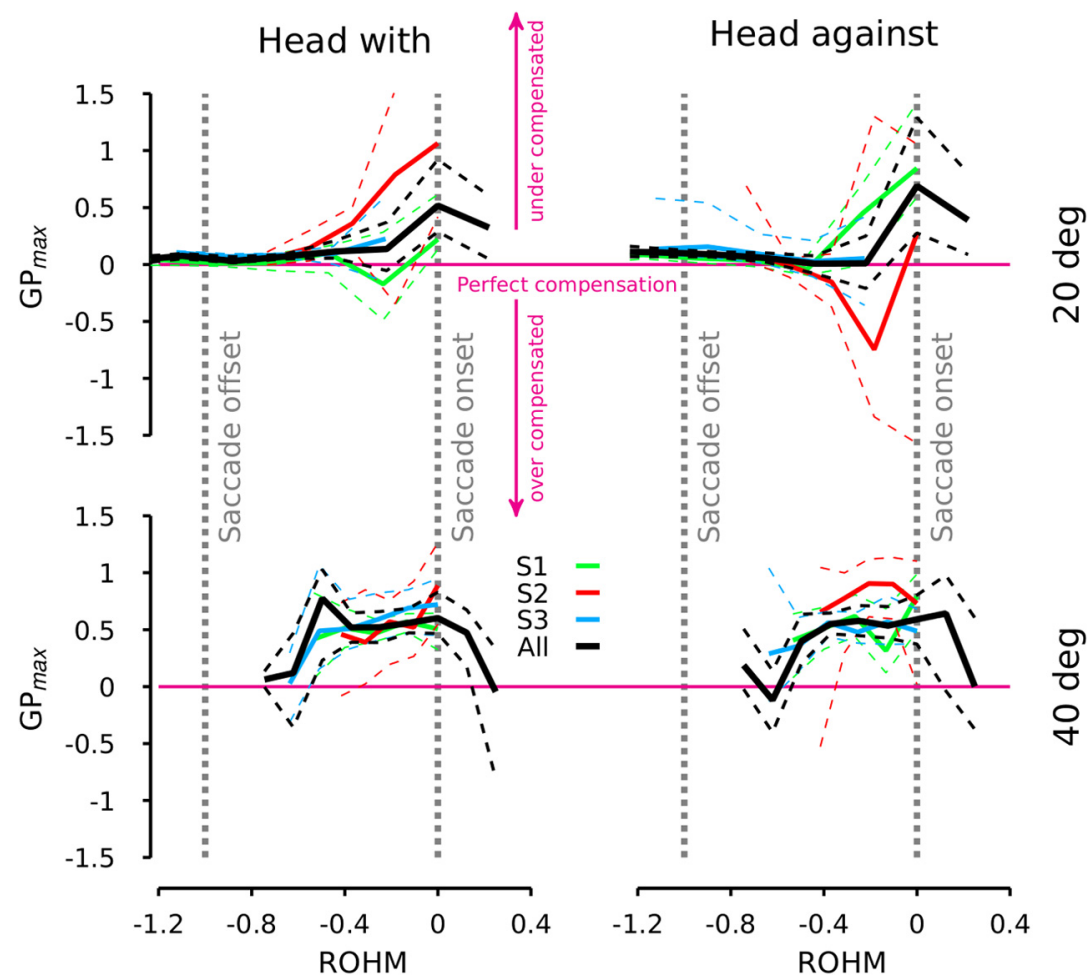

Figure 3. Gain, $G P_{\max }$ at the peak velocity of the perturbation as a function of the ROHM for different subjects. The top row shows the evolution of $G P_{\max }$ for $20\left(40^{\circ}\right.$, bottom) target jumps, all latencies pooled together ( $50 \mathrm{~ms}<$ latency $\left.<300 \mathrm{~ms}\right)$. Thick solid black lines represent the average of $G P_{\text {max }}$ for all the subjects pooled together. Thick solid colored lines represent the average of $G P_{\max }$ for each subject. Dashed lines represent the $95 \%$ confidence interval of the different averaged $G P_{\max }$. The vertical dashed gray lines represent the onset (right line) and the offset (left line) of the saccade.

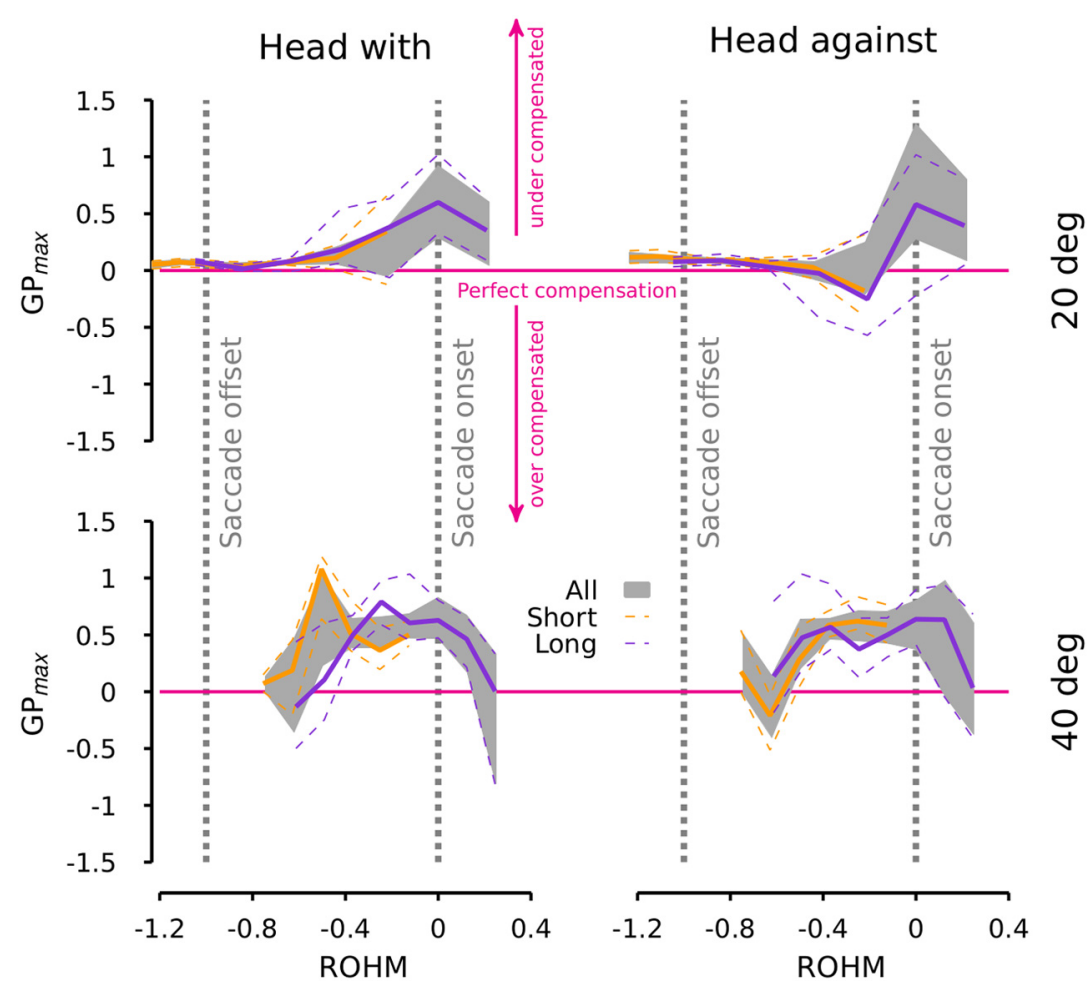

Figure 4. Gain $G P_{\max }$ at the peak velocity of the perturbation as a function of the ROHM for different saccade latencies. Same layout as in Figure 3. The gray area represents the $95 \%$ confidence interval of $G P_{\max }$ for all latencies pooled together. Solid orange lines represent the average of $G P_{\max }$ for saccade latencies shorter than $110 \mathrm{~ms}$. Solid purple lines represent the average of $G P_{\text {max }}$ for saccade latencies larger than $110 \mathrm{~ms}$. Dashed lines represent the $95 \%$ confidence interval of the different averaged $G P_{\max }$. 
cause the eye controller would have no information about how the perturbation might have affected gaze. Therefore, in the case of separate eye and head controllers, the perturbation would generate a final gaze error equal to the amplitude of the chair rotation. As we demonstrated that the gaze accuracy is insensitive to the perturbation, our experiment rejects a control structure based on separate eye and head controllers without gaze feedback, as has also been concluded by others based on other data (Choi and Guitton, 2006; Boulanger et al., 2012).

\section{VOR suppression and gaze feedback}

Traditionally, the VOR gain is computed as the ratio between a change of eye velocity and a change of head velocity during a head perturbation, e.g., as the ratio of the peak eye to peak head velocity change (Cullen et al., 2004), or the ratio of eye to head velocity changes with a time shift $(\sim 6 \mathrm{~ms})$ between them to account for the inherent delayed response of the VOR sensors (Tabak et al., 1996). Sylvestre and Cullen (2006) showed a modulation of brainstem premotor excitatory and inhibitory burst neuron activity when the head was perturbed midflight during head-free saccades. They reported two effects of the perturbation on the activity of the burst neurons: one of short latency (demonstrating a short-path connection between vestibular sensors and burst neurons) and the other of longer latency (demonstrating that burst neurons are inside a feedback loop). Subsequently, Choi and Guitton (2009) showed that neurons on the SC motor map decrease their firing rate with an $\sim 10 \mathrm{~ms}$ latency in response to head brakes. These results confirmed that both direct (via vestibular neurons) and indirect (via collicular neurons) inputs to the burst neurons could modify their discharge at latencies just a little longer than direct vestibular inputs to motoneurons (the classic VOR). Because a head perturbation affects the activity of burst neurons inside the feedback loop, the ratio between eye and head velocities during a gaze shift represents the combined action of the VOR and the feedback loop. Thus, gain ratios demonstrate a VOR modulation during gaze shifts, but they cannot be used to compute a pure VOR suppression factor (and thus an isolated VOR gain) because the perturbation also modulates the neural activity inside the gaze feedback loop. Therefore, both the suppression factor and the gaze-control feedback loop gain must be known to analyze the temporal changes in the gaze trajectory linked to a perturbation, because their action is combined during a gaze saccade. This could explain why there are idiosyncratic differences between subjects in our experiment and in those of others (Cullen et al., 2004), because different subjects could have different VOR gains and different gaze feedback gains. Nevertheless, the combined action of both the VOR and the gaze feedback loop would ensure that gaze shifts ended on target despite the head perturbation, as shown in Table 1 . To summarize, when a gaze trajectory remains unaltered by a perturbation, it reveals that the VOR gain is equal to 1 (because the feedback loop is not fast enough to correct the perturbation on-the-fly). However, when the trajectory of the gaze saccade is altered by the perturbation, the change of trajectory is a reflection of an imperfect VOR gain (the larger the gaze change, the smaller the VOR gain) but the ratio between eye and head velocities cannot be used to extract either the time course of the VOR gain or the gaze feedback gain because their actions are combined.

\section{VOR modulation as a function of gaze amplitude}

Our results, combined with previous observations (Tabak et al., 1996; Cullen et al., 2004) suggest that the VOR is modulated by the gaze shift amplitude, as incorporated in a recent model by
Daye et al. (2014). The VOR cannot be modulated by a head command alone, because the head was not moving actively during our experiment. However, several studies have shown that neck muscles are activated during stimulation of the superior colliculus (Corneil et al., 2002; Rezvani and Corneil, 2008) or the frontal eye fields (Corneil et al., 2010), even when the monkey does not make a head movement. Other studies, using neck muscles recordings with EMG in humans showed tonic and phasic neck muscles activity in absence of head movement (AndréDeshays et al., 1988, 1991). Therefore, another possibility is that an internal signal planning, but not giving rise to, a head movement could also modulate the VOR gain. This signal might decrease the gain of the VOR. However, this would assume that the priming signal was a function of both the planned head and eye movement, because the VOR should only be suppressed when the eye and head move together. Finally, it seems unlikely that a head command (and its associated corollary discharge) too small to generate a head movement would be sufficient to attenuate the saccadic burst generator such that it would compensate for an unexpected perturbation. Thus, it is more parsimonious to assume the gaze command itself decreases the VOR gain though this hypothesis must be addressed experimentally. This could be done with EMG recordings of the neck muscles during an experiment that would compare passive head-on-body rotations as in Pelisson et al. (1988) and whole-body rotation as in this experiment to express what is the influence of the neck command on the modulation of the VOR and the control of the gaze trajectory. Finally, recording the activity of the different cell types carrying vestibular signals during both the Pelisson et al. (1988) task and our task would help to decipher how much of a role VO cells play in the modulation of the vestibulo-ocular reflex per se (for review, see Cullen, 2011, 2012 describing the role of the different vestibular cell types). In addition, these recordings could give insights on how much the VO cells are modulated by neck afference.

\section{Conclusion}

The VOR gain is suppressed during large gaze shifts by a gaze command, and the gaze trajectory arrives on target because of compensatory actions by a gaze feedback controller, which operates whether the head is still or moving. Thus, we conclude that even when no head movement is planned the brain uses feedback control of gaze and not the eye alone. At the same time, this gaze command acts to suppress the VOR if the head should be perturbed by an imposed movement during the initial saccade. Presumably, other sources of feedback, e.g., from neck proprioceptors, would also be used for control of gaze if the head and torso were affected independently.

\section{References}

André-Deshays C, Berthoz A, Revel M (1988) Eye-head coupling in humans. Exp Brain Res 69:399-406. Medline

André-Deshays C, Revel M, Berthoz A (1991) Eye-head coupling in humans: II. Phasic components. Exp Brain Res 84:359-366. Medline

Boulanger M, Galiana HL, Guitton D (2012) Human eye-head gaze shifts preserve their accuracy and spatiotemporal trajectory profiles despite long-duration torque perturbations that assist or oppose head motion. J Neurophysiol 108:39-56. CrossRef Medline

Choi WY, Guitton D (2006) Responses of collicular fixation neurons to gaze shift perturbations in head-unrestrained monkey reveal gaze feedback control. Neuron 50:491-505. CrossRef Medline

Choi WY, Guitton D (2009) Firing patterns in superior colliculus of headunrestrained monkey during normal and perturbed gaze saccades reveal short-latency feedback and a sluggish rostral shift in activity. J Neurosci 29:7166-7180. CrossRef Medline 
Collewijn H, Smeets JB (2000) Early components of the human vestibuloocular response to head rotation: latency and gain. J Neurophysiol 84: 376-389. Medline

Corneil BD, Olivier E, Munoz DP (2002) Neck muscle responses to stimulation of monkey superior colliculus: I. Topography and manipulation of stimulation parameters. J Neurophysiol 88:1980-1999. Medline

Corneil BD, Elsley JK, Nagy B, Cushing SL (2010) Motor output evoked by subsaccadic stimulation of primate frontal eye fields. Proc Natl Acad Sci U S A 107:6070-6075. CrossRef Medline

Crane BT, Viirre ES, Demer JL (1997) The human horizontal vestibuloocular reflex during combined linear and angular acceleration. Exp Brain Res 114:304-320. CrossRef Medline

Cullen KE (2011) The neural encoding of self-motion. Curr Opin Neurobiol 21:587-595. CrossRef Medline

Cullen KE (2012) The vestibular system: multimodal integration and encoding of self-motion for motor control. Trends Neurosci 35:185-196. CrossRef Medline

Cullen KE, Huterer M, Braidwood DA, Sylvestre PA (2004) Time course of vestibuloocular reflex suppression during gaze shifts. J Neurophysiol 92: 3408-3422. CrossRef Medline

Daye PM, Optican LM, Blohm G, Lefèvre P (2014) Hierarchical control of two-dimensional gaze saccades. J Comput Neurosci 36:355-382. CrossRef Medline

Freedman EG (2001) Interactions between eye and head control signals can account for movement kinematics. Biol Cybern 84:453-462. CrossRef Medline

Freedman EG (2008) Coordination of the eyes and head during visual orienting. Exp Brain Res 190:369-387. CrossRef Medline

Freedman EG, Cecala AL (2008) Oblique gaze shifts: head movements reveal new aspects of component coupling. Prog Brain Res 171:323-330. CrossRef Medline

Good PI (2006) Resampling methods: a practical guide to data analysis, Ed 3. Boston: Birkhäuser.

Goossens HH, Van Opstal AJ (1997) Human eye-head coordination in two dimensions under different sensorimotor conditions. Exp Brain Res 114: 542-560. CrossRef Medline

Grossman GE, Leigh RJ, Abel LA, Lanska DJ, Thurston SE (1988) Frequency and velocity of rotational head perturbations during locomotion. Exp Brain Res 70:470-476. Medline

Guitton D, Volle M (1987) Gaze control in humans: eye-head coordination during orienting movements to targets within and beyond the oculomotor range. J Neurophysiol 58:427-459. Medline

Guitton D, Munoz DP, Galiana HL (1990) Gaze control in the cat: studies and modeling of the coupling between orienting eye and head movements in different behavioral tasks. J Neurophysiol 64:509-531. Medline

Kardamakis AA, Moschovakis AK (2009) Optimal control of gaze shifts. J Neurosci 29:7723-7730. CrossRef Medline

Kardamakis AA, Grantyn A, Moschovakis AK (2010) Neural network simulations of the primate oculomotor system: V. Eye-head gaze shifts. Biol Cybern 102:209-225. CrossRef Medline

Lanman J, Bizzi E, Allum J (1978) The coordination of eye and head movement during smooth pursuit. Brain Res 153:39-53. CrossRef Medline

Laurutis VP, Robinson DA (1986) The vestibulo-ocular reflex during human saccadic eye movements. J Physiol 373:209-233. Medline

Lefèvre P, Galiana HL (1992) Dynamic feedback to the superior colliculus in a neural network model of the gaze control system. Neural Netw 5:871890. CrossRef

Lefèvre P, Bottemanne I, Roucoux A (1992) Experimental study and modeling of vestibulo-ocular reflex modulation during large shifts of gaze in humans. Exp Brain Res 91:496-508. Medline

Pelisson D, Prablanc C, Urquizar C (1988) Vestibuloocular reflex inhibition and gaze saccade control characteristics during eye-head orientation in humans. J Neurophysiol 59:997-1013. Medline

Pulaski PD, Zee DS, Robinson DA (1981) The behavior of the vestibuloocular reflex at high velocities of head rotation. Brain Res 222:159-165. CrossRef Medline

Rezvani S, Corneil BD (2008) Recruitment of a head-turning synergy by low-frequency activity in the primate superior colliculus. J Neurophysiol 100:397-411. CrossRef Medline

Schwarz U, Busettini C, Miles FA (1989) Ocular responses to linear motion are inversely proportional to viewing distance. Science 245:1394-1396. CrossRef Medline

Snyder LH, King WM (1992) Effect of viewing distance and location of the axis of head rotation on the monkey's vestibuloocular reflex: I. Eye movement responses. J Neurophysiol 67:861-874. Medline

Sylvestre PA, Cullen KE (2006) Premotor correlates of integrated feedback control for eye-head gaze shifts. J Neurosci 26:4922-4929. CrossRef Medline

Tabak S, Smeets JB, Collewijn H (1996) Modulation of the human vestibuloocular reflex during saccades: probing by high-frequency oscillation and torque pulses of the head. J Neurophysiol 76:3249-3263. Medline

Tomlinson RD (1990) Combined eye-head gaze shifts in the primate: III. Contributions to the accuracy of gaze saccades. J Neurophysiol 64:18731891. Medline

Tomlinson RD, Bahra PS (1986) Combined eye-head gaze shifts in the primate: II. Interactions between saccades and the vestibuloocular reflex. J Neurophysiol 56:1558-1570. Medline 